\title{
Determining the Frequency Distribution of Ocular Manifestations in Multiple Sclerosis Patients
}

\author{
Mohammad Reza Besharati ${ }^{1}$, Abolfazl Hoseinzadeh ${ }^{1}$, Pouria Yazdian-Anari ${ }^{2,}$, Solmaz Ghorbani Aliabadi ${ }^{1}$, \\ Marzieh Vaghefi ${ }^{2}$, Mehrdad Mansouri ${ }^{2}$
}

1. Department of Ophthalmology, Shahid Sadoughi University of Medical Sciences, Yazd, Iran

2. Student Research Committee, Shahid Sadoughi University of Medical Sciences, Yazd, Iran

*Corresponding Author: Pouria Yazdian-Anari, Student Research Committee, Shahid Sadoughi University of Medical Sciences, Yazd, Iran Email: pouria_yazdian_a@yahoo.com

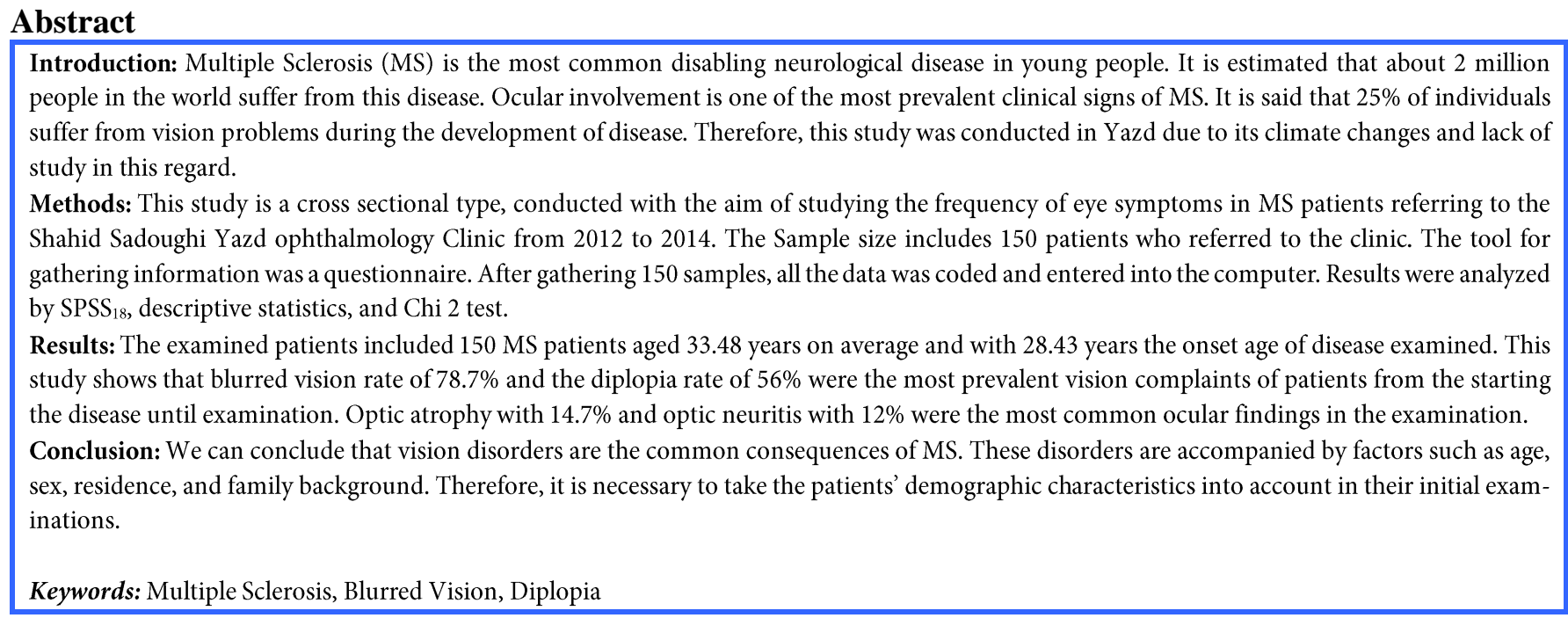

Article History: Received: 2 Jul 2014; Revised: 25 Sep 2014; Accepted: 19 Oct 2014

Cite this article as: Besharati MR, Hoseinzadeh A, Yazdian Anari P, Ghorbani Aliabadi S, Vaghefi M, Mansouri M. Determining the frequency distribution of ocular manifestations in multiple sclerosis patients. Int J Travel Med Glob Health. 2015;3(2):71-4.

\section{Introduction}

Multiple Sclerosis (MS) is the most common disabling neurological disease in young people [1]. It is estimated that about 2 million people in the world suffer from this disease [2]. The cause of MS is not well known but factors such as immune system deficiency, the family history of individuals, familial history, viral infections, mental pressures and stress are thought to contribute to its frequency [2]. Ocular involvement is one of the most prevalent clinical signs of MS [3]. It is seen that $25 \%$ of individuals suffer from vision problems during the development of the disease [4]. Ocular signs include Retrobulbar Neuritis, uveitis, and reduced vision function, nystagmus, INO, diplopia, and Papilitis [5]. Optic neuritis is the most common vision symptom in MS patients which could be the initial manifest of the disease [6].

In the initial stages, MS creates a variety of symptoms. Therefore, vision symptoms could be the first symptoms of the disease and predict other demyelinating problems [7].

So far, no detailed study has been carried out on the importance of vision disorders as the early symptoms of the disease and identification of eye symptoms in the cause of dis- ease improvement phase. Therefore, this study was conducted in Yazd due to its climate changes and lack of study in this regard.

\section{Methods}

This study is a cross sectional type conducted with the aim of studying frequency of eye symptoms in MS patients referring to the Shahid Sadoughi Yazd ophthalmology Clinic from 2012 to 2014.

Inclusion criteria were all the MS patients who referred to the Shahid Sadoughi Yazd Hospital, with their disease confirmed by neurologist. The sample size includes 150 of the patients who referred to this clinic.

The tool for gathering information was a questionnaire. The first section of this questionnaire was the demographic information of patients (sex, age, disease starting age, living place (city or village) and the patient's family history and systemic disease. The second section was related to eye examinations which was covered by ophthalmologist including the visual acuity, slit lamp examination, fundus copy, and external ocular muscles and color vision test by Ishihara. 
After gathering 150 samples, all data were coded and entered into the computer. Results were analyzed by SPSS.18.

\section{Results}

150 MS patients aged 33.48 years on average and with 28.43 years the onset age of disease examined. 24 patients (16\%) were male and 126 patients $(84 \%)$ were female. $92 \%$ of patients live in urban areas and $8 \%$ in rural areas. $16 \%$ of patients had family history of MS and $84 \%$ had no family history.

This study shows that the blurred vision rate with $78.7 \%$ and diplopia with $56 \%$ were the most prevalent vision complaints of the patients from the starting of the disease till examination. Optic atrophy was $14.7 \%$ and optic neuritis with $12 \%$ were the most common ocular findings in the examination (table 1).

Paresthesia and hypoesthesia with $89.3 \%$ frequency and weakness with $76 \%$ frequency were the most neurological symptoms in patients. Migraine was the more prevalent accompanying systemic disease in patients $(9.3 \%)$.

In $42.7 \%$ of the patients, the disease had started with eye symptoms, in $42.7 \%$ with neurological symptoms, and in $14.7 \%$ with eye and neurologic (both) symptoms. The most common symptoms of the first symptom were blurred vision with the frequency of $45.3 \%$ and Paresthesia and Hyoposthesia with the frequency of $44 \%$.

Diplopia was not frequent in the group aged under 30 compared to the ones over 30 . This was statistically significant. Nystagmus was most prevalent in patients under 30 years. The disorder was not observed in people above 30 years. Uhthoff's phenomenon was significantly observed in women and disc atrophy was most frequent in men.

Nystagmus and optic atrophy were more frequent in patients who aged 15-24 years at the start of the disease. This difference was statistically significant. In patients who aged 25-34 years at the beginning, impaired color vision was more prevalent.

Optic neuritis, impaired color vision disorder and oculomotor disorders were significantly more prevalent in rural patients than urban areas.

In patients who had a family background of MS, the INO symptom was significantly more prevalent than patients with no family history of the disease.

Among the patients in this study, 24\% of them had significant eye symptoms such as optic neuritis, retrobulbar neuritis, uveitis, optic disc atrophy, papilitis, and oculomotor disorders. These symptoms were significantly more prevalent in male patients who lived in rural areas.

\section{Discussion}

The general purpose of this study was determining the frequency of the distribution of ocular and neurological symptoms in MS patients and its relationship with demographic characteristics and disease characteristics. In a study conducted by Alroughani R, 736 patients in the study aged 29.5 years on average and the average onset age of disease start was 26.87 years [8]. In a study by El-Salem in Jordan, the average age of $224 \mathrm{MS}$ patients was 29.3 years [9]. MS outbreak starts in ages 20-40 years [2]. In our study also, the average age of patients was 33.48 years and the average onset disease start age was 28.43 years, which was similar to conducted studies.

Table 1. Prevalence and percentage of eye demonstration, neurological symptoms, and systemic diseases associated

\begin{tabular}{|c|c|c|c|c|c|c|c|c|}
\hline Systemic Diseases Associated & Percent & Number & Neurological Symptoms & Percent & Number & Eye Demonstration & Percent & Number \\
\hline Thyroid disease & 8 & 12 & Paresthesia and Hyoposthesia & 89.3 & 134 & blurred vision & 78.7 & 118 \\
\hline Hyperlipidemia & 2.7 & 4 & Weakness & 76 & 114 & Diplopia & 56 & 84 \\
\hline Hypertension & 2.7 & 4 & depression & 58.7 & 88 & ocular pain & 37.3 & 56 \\
\hline \multirow[t]{7}{*}{ Migraine } & 9.3 & 14 & Bladder dysfunction & 33.3 & 50 & optic neuritis & 21.4 & 32 \\
\hline & & & Fatigue & 82 & 123 & Uveitis & 0 & 0 \\
\hline & & & Dizziness & 46.7 & 70 & INO & 5.3 & 8 \\
\hline & & & Sexual Dysfunction & 29.3 & 44 & Nystagmus & 4 & 6 \\
\hline & & & Lhermitte's sign & 22.7 & 34 & disc atrophy & 14.7 & 22 \\
\hline & & & & & & Dyschromatopsia & 9.3 & 14 \\
\hline & & & & & & oculomotor disorder & 2.7 & 4 \\
\hline
\end{tabular}

Table 2. Relation and prevalence of eye demonstration with age groups of study

\begin{tabular}{|c|c|c|c|c|}
\hline $\begin{array}{l}\text { Age } \\
\text { Eye Demonstration }\end{array}$ & $<30$ & $30-39$ & $>40$ & P-Value \\
\hline Blurred vision & $42(80.8 \%)$ & $42(77.8 \%)$ & $34(77.3 \%)$ & 0.899 \\
\hline Diplopia & $18(34.6 \%)$ & $34(63 \%)$ & $32(72.7 \%)$ & 0.0001 \\
\hline Ocular pain & $14(26.9 \%)$ & $24(44.4 \%)$ & $18(40.9 \%)$ & 0.148 \\
\hline Uhthoff's phenomenon & $14(26.9 \%)$ & $10(18.5 \%)$ & $10(22.7 \%)$ & 0.586 \\
\hline Optic neuritis & $18(34.5 \%)$ & $8(14.8 \%)$ & $6(13.6 \%)$ & 0.135 \\
\hline Uveitis & $0(0 \%)$ & $0(0 \%)$ & $0(0 \%)$ & - \\
\hline INO & $2(3.8 \%)$ & $4(7.4 \%)$ & $2(4.5 \%)$ & 0.690 \\
\hline Nystagmus & $6(11.5 \%)$ & $0(0 \%)$ & $0(0 \%)$ & 0.003 \\
\hline Disc atrophy & $10(19.2 \%)$ & $10(18.5)$ & $2(4.5 \%)$ & 0.078 \\
\hline Dyschromatopsia & $4(7.7 \%)$ & $8(14.8 \%)$ & $2(4.5 \%)$ & 0.194 \\
\hline Oculomotor disorder & $0(0 \%)$ & $2(3.7 \%)$ & $2(4.5 \%)$ & 0.325 \\
\hline
\end{tabular}


Table 3. Relation and prevalence of eye demonstration with location of life

\begin{tabular}{lccc}
\hline $\begin{array}{l}\text { Location } \\
\text { Eye Demonstration }\end{array}$ & Village & City & P-Value \\
\hline Blurred vision & $10(83.3 \%)$ & $108(78.3 \%)$ & 1 \\
Diplopia & $6(50 \%)$ & $78(56.5 \%)$ & 0.622 \\
Ocular pain & $2(16.7 \%)$ & $54(39.1 \%)$ & 0.212 \\
Optic neuritis & $4(33.3 \%)$ & $14(10.1 \%)$ & 0.039 \\
Retro bulbar neuritis & $2(16.7 \%)$ & $8(5.8 \%)$ & 0.184 \\
Uveitis & $0(0 \%)$ & $0(0 \%)$ & - \\
INO & $0(0 \%)$ & $8(5.8 \%)$ & 1 \\
Nystagmus & $0(0 \%)$ & $6(4.3 \%)$ & 1 \\
Disc atrophy & $4(33.3 \%)$ & $18(13 \%)$ & 0.078 \\
Dyschromatopsia & $4(33.3 \%)$ & $10(7.2 \%)$ & 0.016 \\
Papilitis & $0(0 \%)$ & $4(2.9 \%)$ & 1 \\
oculomotor disorder & $2(16.7 \%)$ & $2(1.4 \%)$ & 0.032 \\
\hline
\end{tabular}

Table 4. Relation and prevalence of eye demonstration familial history

\begin{tabular}{lccc}
\hline $\begin{array}{l}\text { Familial History } \\
\text { Eye Demonstration }\end{array}$ & Without & With & P-Value \\
\hline Blurred vision & $102(81 \%)$ & $16(66.7 \%)$ & 0.117 \\
Diplopia & $70(55.6 \%)$ & $14(58.3 \%)$ & 0.802 \\
Ocular pain & $48(38.1 \%)$ & $8(33.3 \%)$ & 0.658 \\
Optic neuritis & $30(23.8 \%)$ & $2(8.3 \%)$ & 0.739 \\
Uveitis & $0(0 \%)$ & $0(0 \%)$ & - \\
INO & $4(3.2 \%)$ & $4(16.7 \%)$ & 0.023 \\
Nystagmus & $4(3.2 \%)$ & $2(8.3 \%)$ & 0.246 \\
\hline Disc atrophy & $20(15.9 \%)$ & $2(8.3 \%)$ & 0.53 \\
\hline Dyschromatopsia & $10(7.9 \%)$ & $4(16.7 \%)$ & 0.242 \\
\hline Oculomotor disorder & $4(3.2 \%)$ & $0(0 \%)$ & 1 \\
\hline
\end{tabular}

In this study, the ratio of female to male patients was more than 5 to 1 . The outbreak of MS in women is twice as men [2]. Study of Miller et al. showed that this ratio can be different in various geographical locations and female to male ratio could be 2 to 1 as an indication of the influence of genetic and environmental factors [10]. In a study by El-Salem in Jordan, which is similar to Iran in view of geographical location, female to male ratio exceeds 6 to 1 relatively similar to our study $[9,10]$.

In this study, $92 \%$ of the patients lived in urban areas and $8 \%$ lived in rural areas. In Granier's study, modern living style, industrialization, and living in urban areas, were considered as risk factors in the outbreak of MS [11]. In a study conducted in the United States, the rate of disease in urban areas was four times more compared to rural areas. MS outbreak was more prevalent in city areas in Australia $[10,11]$.

In this study $16 \%$ of the patients had a family history of MS. In a study by El-Salem, $9.4 \%$ of 224 patients had a family history of MS [9]. In a study by Fernandez-Perez et al., 13 out of 308 examined patients (7.47\%) had a family ground of MS [12]. The reason of this rate in our study may be attributed to the small size of sample and high rates of family marriages in Yazd.

Optic neuritis is the most common vision symptom in MS and can be sometimes the first symptom of the disease [6]. The outbreak of optic neuritis was $20 \%$ according to a study by El-Salem [9].

In our study $56 \%$ of the patients had diplopia. In a similar study conducted by Jan M Roodhoft, $17.9 \%$ of 284 patients had diplopia. The reason of this high rate in our study may be attributed to the small size of sample. In our study, diplopia had the most rate of occurrence between the ages 30 39 years old.

In our study also, optic neuritis was the most common vision symptom during examination (frequency of $12 \%$ ). Blurred vision as the most common consequence of optic neuritis with a frequency of $78 \%$ was the most frequent vision-related complaints of patients from the outbreak through the examination. This was probably due to previous attacks of optic neuritis in patients.

In a study conducted by Aranda E in Spain, the most prevalent clinical symptoms of patients included motor symptoms (paralysis) and sensation symptoms (Paresthesia) [13]. In our study, paresthesia and hypoesthesia ( $89.3 \%$ frequency) were the most common complaint of patients during this disease. In the Hoang PD study, 70\% of 156 MS patients were assessed regarding their muscular strength. Among them $70 \%$ of patients had muscular weakness in one or several groups of muscles [14]. The outbreak of muscular weakness in our study was $76 \%$.

In a study conducted by Sidhomy, the outbreak of this disease was accompanied by neural symptoms (motor and sensation) in $44 \%$, while $20 \%$ of the patients showed optic neuritis [15]. In our study, $42.7 \%$ of the patients reported that the disease outbreak was accompanied by neural symptoms such as paresthesia and hypoesthesia, weakness and vertigo. Also, $42.7 \%$ of the patients reported vision symptoms including blurred vision and double vision in the start of their disease. It was also noticeable that $14.7 \%$ of patients reported that the outbreak of their disease was accompanied by vision 
and neural symptoms (both).

Nystagmus was significantly prevalent in people under 30 , disc atrophy in men, optic neuritis, color impaired vision disorder and oculomotor disorders in patients residing in rural areas and INO in patients with MS family background. Unfortunately, no single study was available to have studied the emergence of vision manifestations based on demographic characteristics.

The disease risk factors included aging below 40 as start of the disease, being male as a risk factor in precaution and positive family background $s$ [1].

Twenty four percent of the patients of the study had significant vision symptoms including optic neuritis, retrobulbar neuritis, uveitis, disc atrophy and oculomotor disorders. These vision symptoms were significantly more prevalent in male individuals. This can be justified based on the fact that being male is a risk factor in the disease precaution [3]. These vision symptoms were significantly more prevailing in patients residing in rural areas.

\section{Conclusion}

Regarding the results of our study and comparing them with other studies we can conclude that vision disorders are the common consequences of MS. These disorders are accompanied by factors such as age, sex, residence, and family background. Therefore, it is necessary to take the patients' demographic characteristics into account in their initial examinations. Vision symptoms are common in the disease outbreak. Therefore, the MS disease should be considered when examining patients who refer with vision complaints such as blurred vision and double vision.

\section{Acknowledgements}

We would like to thank Mr. Ayoubian for his collaboration.

\section{Authors' Contribution}

All authors contributed in all the steps of this study.

\section{Funding/Support}

This study was support by the Shahid Sadoughi University of Medical Sciences.

\section{Financial Discloser}

Not declared.

\section{References}

1. Frohman EM, Frohman TC, Zee DS, McColl R, Galetta S. The neuroophthalmology of multiple sclerosis. The Lancet Neurology. 2005;4(2):111-21.

2. Greenberg D, Aminoff M, Simon R. Clinical Neurology 8/E: McGraw Hill Professional; 2012

3. Sakai RE, Feller DJ, Galetta KM, Galetta SL, Balcer LJ. Vision in multiple sclerosis: the story, structure-function correlations, and models for neuroprotection. Journal of neuro-ophthalmology: the official journal of the North American Neuro-Ophthalmology Society. 2011;31(4):362-73.

4. Adams R, Victor M, Ropper A. Principle of neurology 7th ed. McGrawhill; 2005.
5. Fischer LG. The ocular manifestations of multiple sclerosis. Journal of the American Optometric Association. 1977;48(12):1511-5.

6. Chen L, Gordon LK. Ocular manifestations of multiple sclerosis. Current opinion in ophthalmology. 2005;16(5):315-20.

7. JM R. Ocular problems in early stages of multiple sclerosis. Bull soc belge ophtalmol. 2009;313:65-8.

8. Alroughani R, Ahmed SF, Al-Hashel J. Demographics and clinical characteristics of multiple sclerosis in Kuwait. European neurology. 2014;72(3-4):181-5.

9. El-Salem K, Al-Shimmery E, Horany K, Al-Refai A, Al-Hayk K, Khader Y. Multiple sclerosis in Jordan: A clinical and epidemiological study. Journal of neurology. 2006;253(9):1210-6.

10. Miller DH, Fazekas F, Montalban X, Reingold SC, Trojano M. Pregnancy, sex and hormonal factors in multiple sclerosis. Multiple sclerosis. 2014;20(5):527-36.

11. Granieri E. Exogeneous factors in the aetiology of multiple sclerosis Journal of neurovirology. 2000;6 Suppl 2:S141-6.

12. Fernandez-Perez MJ, Barakat O, Garcia-Moreno JM, Lucas M, Navarro G, Gata JM, et al. [Clinical features of familial multiple sclerosis in Spain]. Revista de neurologia. 1999;29(8):693-6.

13. Anton Aranda E, Martinez-Lage JM, Maravi Petri E, Gallego Cullere J, de Castro P, Villanueva Eusa JA. [Epidemiology and clinical evolutionary aspects of multiple sclerosis in Navarra]. Neurologia (Barcelona, Spain). 1991;6(5):160-9.

14. Hoang PD, Gandevia SC, Herbert RD. Prevalence of joint contractures and muscle weakness in people with multiple sclerosis. Disability and rehabilitation. 2014;36(19):1588-93.

15. Sidhom Y, Damak M, Riahi A, Hizem Y, Mrissa R, Mhiri C, et al. Clinical features and disability progression in multiple sclerosis in Tunisia: do we really have a more aggressive disease course? Journal of the neurological sciences. 2014;343(1-2):110-4. 